\title{
Active flow control of a diffusing S-duct
}

\author{
Yahya Hamza Shanan ${ }^{1^{*}}$ \\ ${ }^{1}$ Department of Aeronautical Engineering, Defense University, College of Engineering, ETHIOPIA \\ *Corresponding Author's e-mail: yahia22999@gmail.com, Tel: +249-912155433 \\ ORCID iD: http://orcid.org/0000-0003-0715-4558
}

\begin{abstract}
Realization of the S-duct benefits requires scrutiny of certain phenomena such as swirls, secondary flows, and flow distortion creation which are related to the S-duct due to its physical curvature. The first phase of the study investigated the flow field inside the S-duct. NASA Glenn research center's S-duct was adopted in this study. Geometry was created in SOLIDWORKS and mesh was done using ICEM CFD. Numerical analysis was carried out in ANSYS Fluent software. A turbulence model named $\mathrm{k} \omega$-SST was incorporated. Boundary conditions were set so as to match the experimental test done by NASA Glenn research center. Computational results showed a significant agreement with the experiment. The results also affirmed the presence of the secondary flow and flow distortion at the aerodynamic interface plane. The second phase of the study investigated the ability to control the flow and reduce the flow distortion on the engine fan face. Results showed a $10 \%$ flow distortion reduction and the secondary flow severity decreased by $16.5 \%$ on the aerodynamic interface plane (AIP).
\end{abstract}

Keywords: Active Flow Control (AFC), Aerodynamic Interface Plane (AIP), Flow Distortion, S-duct

DOI: http://dx.doi.org/10.4314/ijest.v12i2.1

\section{Introduction}

Serpentine duct or (S-duct) is a new configuration that provides the necessary air to an internally mounted engine (within the fuselage) through an externally mounted inlet. The idea of adopting such configuration stems from its several benefits represented in drag reduction, minimization of engine noise, fuel consumption reduction (Gridley and Walker, 1996). The important role that the UAV plays nowadays justifies the special interest in such configuration due to its stealth capability. This stealth capability stems from submerging the engine into the fuselage in contrast to the conventional configurations in order to prevent the radar beams from directly hitting the engine face. Furthermore, S-duct has recently been incorporated in a new technology adopted by NASA named "Boundary Layer Ingesting Duct" mainly used in NASA/Boing Blended Wing Body "BWB” (Harrison et al., 2013 Angela, 2003, Lewis et al, 2008, Gorton et al., 2004; Giuliani et al., 2014, Onkar et al., 2009, Bobby and Brian, 2004). However, in order to realize the benefits of the S-duct, we had to firstly address particular phenomena such as swirls, flow separation, and secondary flow. These phenomena are inherently existed in this configuration due to its physical curvature and cross-sectional diffusion (Aslan, 2017). More specifically, the secondary flow has the ability to drive the low momentum boundary layers from the periphery of the duct towards the bottom of the duct's second turn creating an area of total pressure deficit on the AIP (Bruce et al., 1992). Total pressure loss and flow non-uniformity severely affect the compressor blades where more work is required from the compressor on the low total pressure (distorted) compared to the higher total pressure (undistorted) (Clinton, 1972).

The study aimed to investigate the nature of the flow-field inside the S-duct as well as clearly understanding the secondary flow and flow distortion creation mechanism. Furthermore, the study investigated the ability to address the secondary flow in an attempt to minimize the flow distortion on the AIP through incorporating a flow control method named as "Active Flow Control". The following flow diagram shows all the steps carried out in this study from the first step of understating the flow distortion mechanism based upon previous investigations, up to the final results of the AFC method implementation. 


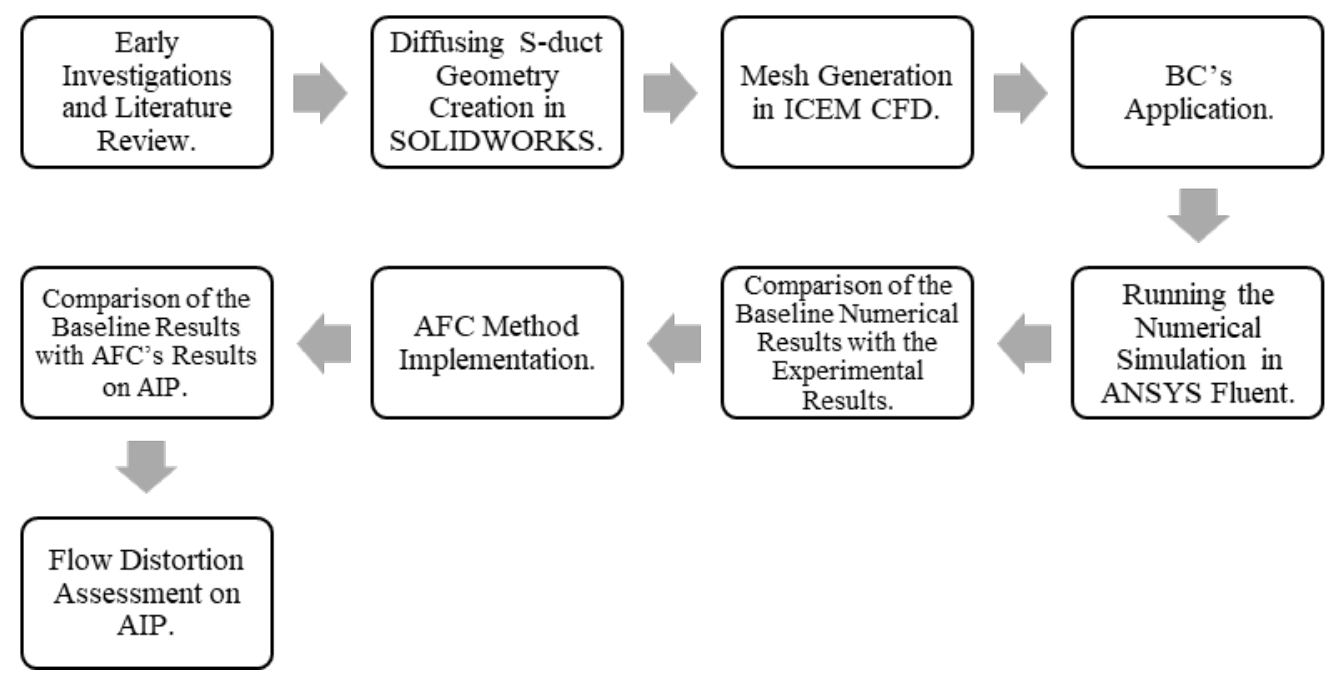

Figure 1. A flow diagram shows the steps carried out in the study

\section{Methodology}

From previous studies it was found that flow through the engine's duct is being controlled either by using simply physical objects in a form of vane-type vortex generators which is named as Passive Flow Control (PFC) method (Bernard, 1992; Bruce et al., 1993; Bruce et al., 1994; Shams et al., 2016), or by using embedded jets, Active Flow Control (AFC) method, in order to counter the adverse flow driven by the strong secondary flows. Early investigations found that PFC had the ability to re-energize the flow in areas of strong adverse pressure gradients which resulted in a reduction of the secondary flow strength and a flow separation delay (original design of Boeing 727). However, apart from the additional weight by the PFC due to the vane-type vortex generators installation, AFC method has many advantages over the PFC method since the arrays of jets are adaptable to any flight condition and can be turned on, adjusted, or turned off as needed (Brian et al., 2006). AFC method could also result in a highly adaptive and efficient system capable of maintaining low-distortion flow when coupled with a closed-loop feedback controller and a network of sensors to detect flow conditions as stated by Neal Harrison in his study of controlling the flow through a Boundary Layer Ingesting (BLI) S-duct (Harrison et al., 2013).

2.1 Diffusing s-duct geometry: The S-duct geometry adopted in this study was previously employed by NASA Glenn research center for the purpose of studying the complex turbulent flow phenomena (Wellborn et al., 1993). It was created in this study using SOLIDWORKS according to the given dimensions. Two identical circular arcs were created in the same plane, each of them has a radius of $\mathrm{R}=1.02 \mathrm{~m}$ where $\theta_{\max } / 2=30^{\circ}$ as depicted in Figure 2. The duct's area diffuses along the centerline. The ratio between the outlet and the inlet cross-sectional area is equal to 1.52 . The inlet has a radius of $0.1021 \mathrm{~m}$ where the outlet radius is $0.1257 \mathrm{~m}$.

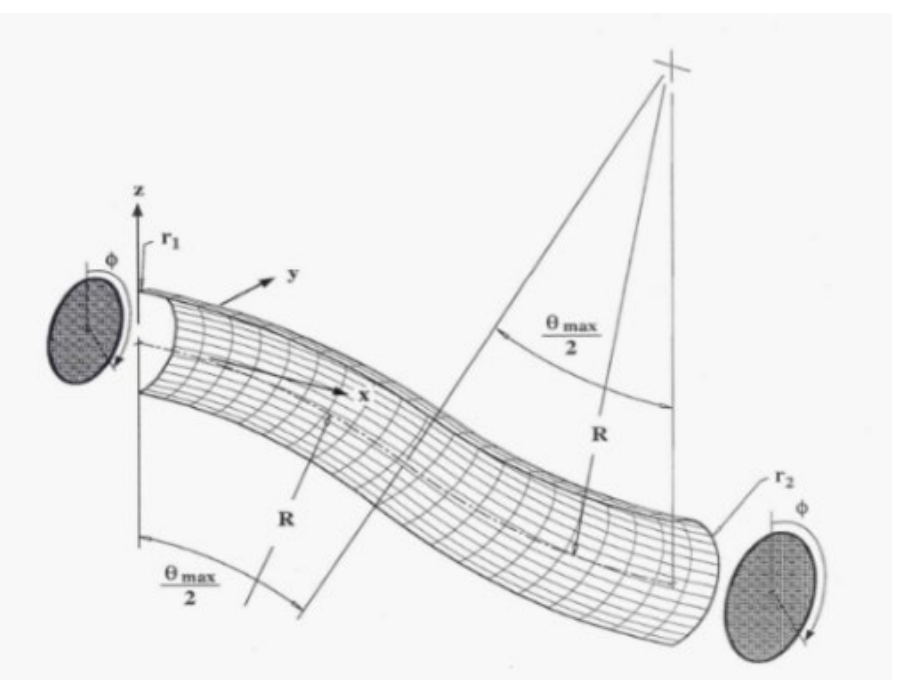

Figure 2. Half shell representation of NASA Glenn's diffusing S-duct (Wellborn et al., 1993)

The flow near the duct curvature was experimentally characterized by the recirculation and flow separation. Therefore, two ducts with constant cross-sectional area and length of $765.75 \mathrm{~mm}$ were attached at the inlet and the outlet of the S-duct to provide an 
ideal location to apply inlet and outlet boundary conditions away from the recirculation zone. The half model was considered not only for the simplicity and computational efficiency purposes, but also because the flow was experimentally approved to be symmetric (Bruce et al., 1992). Figure 3 shows the half model computational domain as the flow goes from the left (computational domain inlet) to the right (computational domain outlet). The figure also shows the diffusion of the duct along the x-axis.

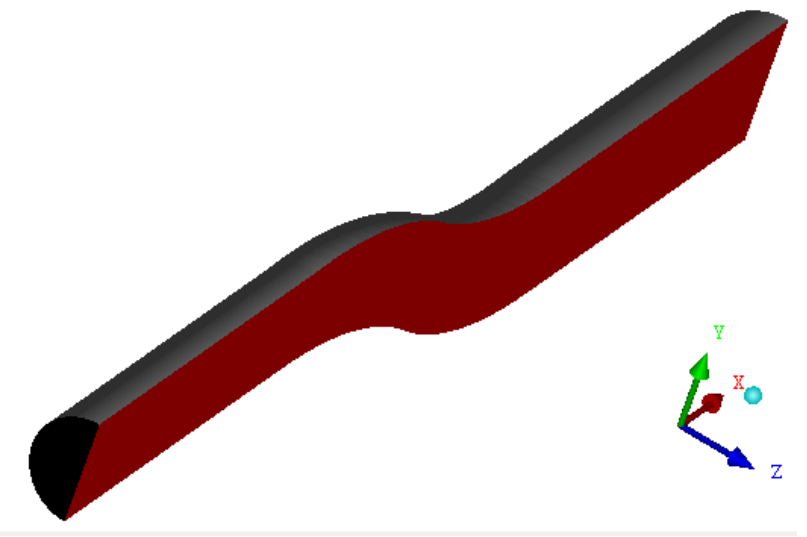

Figure 3. S-duct computational domain

2.2 Mesh creation: The mesh was created in ANSYS ICEM CFD since it provides the user with a high-quality hexahedral mesh (ANSYS ICEM CFD 13., 2014). Capturing the viscous sublayer and the log-layer was very crucial in this study, therefore, an $\mathrm{O}+\mathrm{H}$ grid was employed to attain a very fine near-wall mesh. The grid also captured the duct curvature properly. Nodes near the wall were redistributed and the least quality elements were improved to $70 \%$. $\mathrm{O}+\mathrm{H}$ grid structure is shown in Figure 4. Mesh size details are also shown in Table (1).

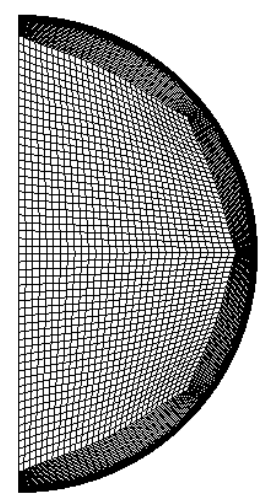

Figure 4. Cross-section view of the $\mathrm{S}-$ duct inlet showing $\mathrm{O}+\mathrm{H}$ grid structure

Table 1. Mesh size details

\begin{tabular}{|c|c|c|}
\hline Nodes & Quads & Hexas \\
\hline $3,677,171$ & 189,540 & $3,592,404$ \\
\hline
\end{tabular}

Boundary conditions were applied so as to match the experimental conditions conducted by NASA Glenn research center. One of the experiment criteria that the flow has to achieve Mach number of 0.6 at the inlet, therefore, 'pressure inlet' boundary condition was set at the computational domain inlet. The walls were set as 'walls' which were chosen to be stationary with no-slip adiabatic condition. The computational domain outlet was set as 'mass flow inlet' whose value also matched the predefined Mach number. The symmetry plane was set as 'symmetry'. Eventually, mesh file was written to Fluent CFD solver.

2.3 Numerical simulation procedure: The CFD flow solver employed in this study was ANSYS Fluent which is capable of solving the three-dimensional Reynolds-Averaged-Navier-Stokes (RANS) equations on an unstructured mesh. The solver employed was steady and able to analyze the compressible flow through the duct. The density was expected to vary along the duct, therefore, a second order accurate density-based solver was employed. k- $\omega$ SST turbulence model was utilized since it was characterized by its suitability for complex flows involving moderate swirl, vortices, and locally transitional flows such as boundary layer separation. It was also more accurate in predicting flow separation than other RANS models (ANSYS Fluent 13, 2015). The case was analyzed using non-dimensional coordinates and non-dimensional flow variables. Five measurement planes were created along the 
duct each of them is referred to using the ratio of the axial distance from the inlet $(\mathrm{x})$ to the inlet diameter $\left(\mathrm{D}_{1}\right)$. The origin is located exactly at the middle of the duct where $\mathrm{x} / \mathrm{D}_{1}=0$. Static and total pressure are expressed in the form of the non-dimensional pressure coefficients as shown in Equations (1) and (2), respectively. Local total pressure is denoted by $p_{o}$ and the local static pressure is denoted by $p$. Reference values of static and total pressure are denoted by $p_{c l}$ and $p_{o, c l}$ respectively.

$$
\begin{gathered}
C_{p}=\frac{p-p_{c l}}{p_{o, c l}-p_{c l}} \\
C_{p o}=\frac{p_{o}-p_{c l}}{p_{o, c l}-p_{c l}}
\end{gathered}
$$

\section{Distortion Descriptors}

Evaluating the level of distortion at the AIP was based upon different measurements obtained from a six-arm distortion probe that measured total pressures in $60^{\circ}$ sections. Five probes located at each arm which represented an angularly equal wedge-shaped section of $60^{\circ}$. Values from these probes were then employed to determine the flow distortion at the AIP. General flow health was evaluated by using the DC (60) $\mathrm{p}_{\mathrm{t}}$ parameter.

$$
D C(60)_{P_{t}}=\frac{P_{t_{\max }}-P_{t_{\min }}}{P_{t_{\max }}-P_{t_{\text {ave }}}}
$$

Examining the ratio of the difference between the average and minimum total pressure and the face dynamic pressure was conducted using the DC (60) q parameter.

$$
D C(60)_{q}=\frac{P_{t_{\text {ave }}}-P_{t_{\min }}}{q_{\text {max }}}
$$

Secondary flow severity evaluated using the SC (60) parameter which characterized the non-axial or transverse flow that could severely harm the compressor blades. $\mathrm{V}_{\mathrm{CF} \text { max }}$ represents the maximum average secondary flow over a $60^{\circ}$ sector while $\mathrm{V}_{\mathrm{CF}}$ min represents the minimum average secondary flow over a $60^{\circ}$ sector and $\mathrm{V}_{\text {ave }}$ represents the average flow velocity at the AIP.

$$
S C(60)=\frac{V_{C F_{\max }}-V_{C F_{\min }}}{V_{\text {ave }}}
$$

\section{Baseline Computational Results}

Computed total pressure contours along the duct at the five measurement planes are presented in Figure 5 as the free stream flow is from left to right. It was noticeable that the flow was uniform, fully developed and free of any transverse velocities at the reference inlet plane (plane A) while the circumferential low-momentum boundary layers started to get larger as indicated in plane B. An area of low total pressure contours was created at the bottom of plane C. This low total pressure was increasing from plane D to plane E due to the secondary flow, creating an area of total pressure deficit at the bottom of the AIP (plane E). 


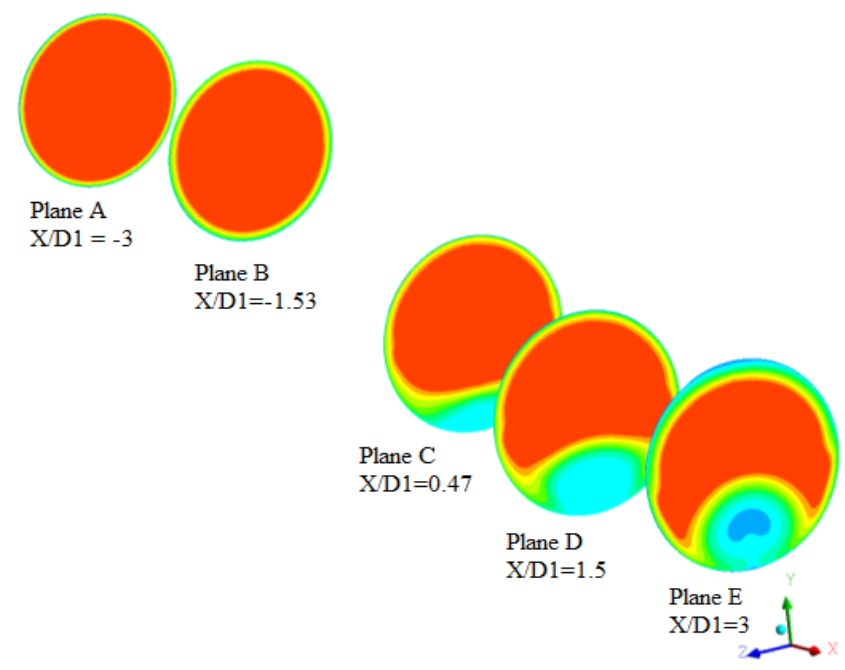

Figure 5. Total pressure coefficient contours at five measurement planes

Streamlines on the symmetry plane depicted in Figure 6 shows a good agreement with the experiment. It was remarkable that the streamlines had taken the same curved shape of the duct's first bend. However, due to the separation, the streamlines were forced to deviate from the curvature of the second bend. The reversed flow region was also noticeable on the second bend of the duct where it began thin and continuously grew until reattachment. The area of the low momentum fluid at the bottom of the duct remained below the duct's centerline even after the reattachment while experimentally the different pressure gradients forced the low momentum flow to deviate above the duct's centerline. This discrepancy between the experimental and computational results was attributed to the failure of the turbulence model to predict this flow phenomenon. The inability of the numerical algorithm to predict the flow deviation above the duct's centerline near the outlet was previously documented by several researchers.

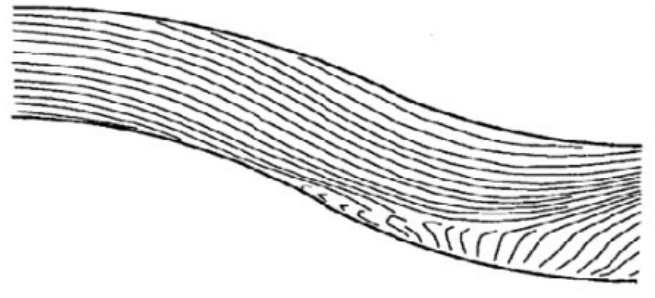

(A)

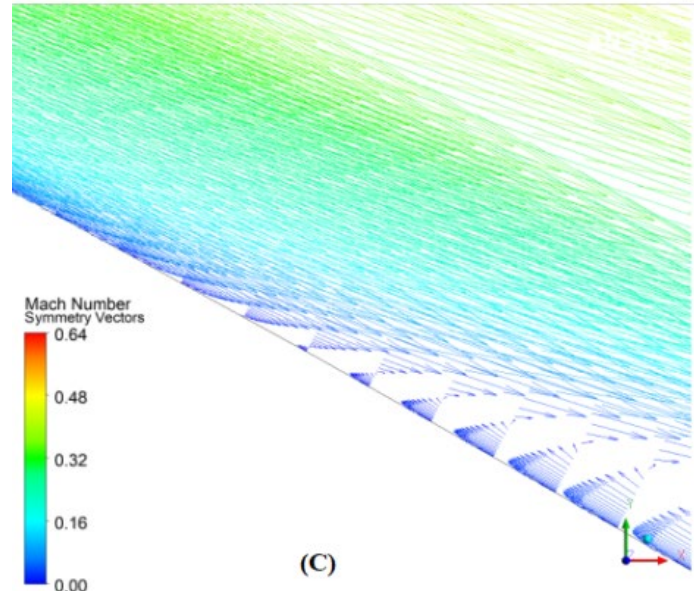

(C)

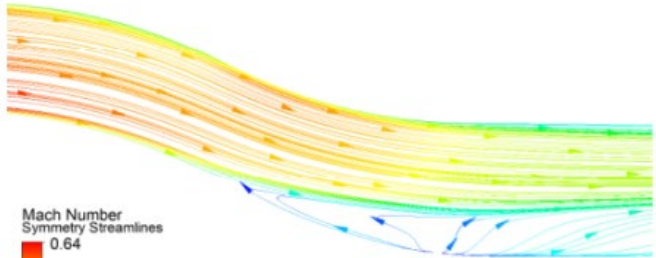

(B)
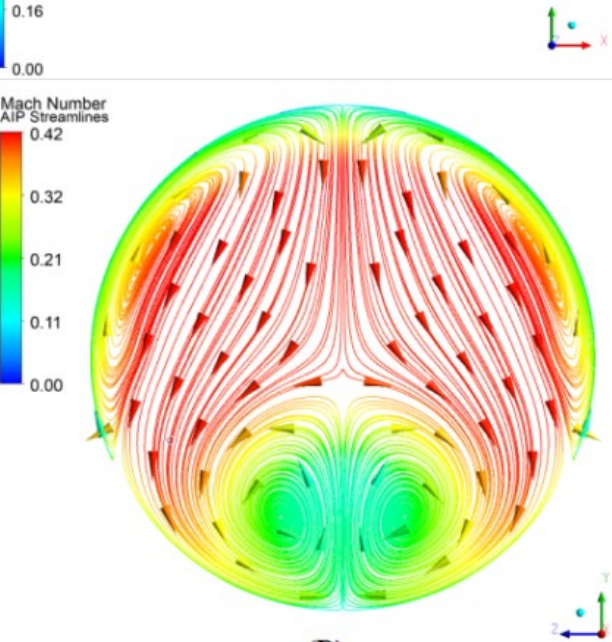

(D)

Figure 6. Mach number streamlines (a) NASA Glenn experimental flow streak-lines (Bruce et al., 1992) (b) Current study symmetry streamlines (c) Boundary layers' separation close up view (d) AIP secondary flows streamlines 
Transverse velocity components and stream-wise vorticity of the AIP are also shown in Figure 6-D. Low-momentum flow streamlines (in green) driven by a strong secondary flow streamlines (in red) were collected at the bottom. As a result, a pair of counter-rotating vortices obviously located on the lower half of the duct evolved. These vortices continuously try to drive the lowmomentum fluid from the bottom towards the center of the duct. A great similarity between the computed and experimental Mach number contours was attained on the AIP. Figure 7 is a comparison between Mach number contours on AIP and the experimental results done by NASA Glenn as well as a previous computational results carried out by Fiola (2013) (Washington University in St. Louis). The figure shows the flow non-uniformity on the AIP where a large area of low momentum flows is created at the bottom. Experimental results showed the low-momentum flow was converted above the centerline of the duct while the current study failed to predict this convection and showed the low-momentum flow below the duct centerline.

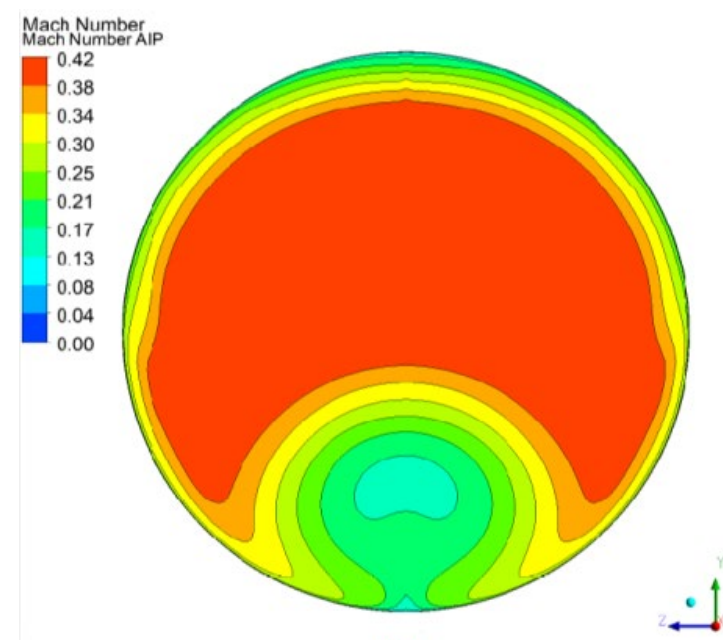

(A)
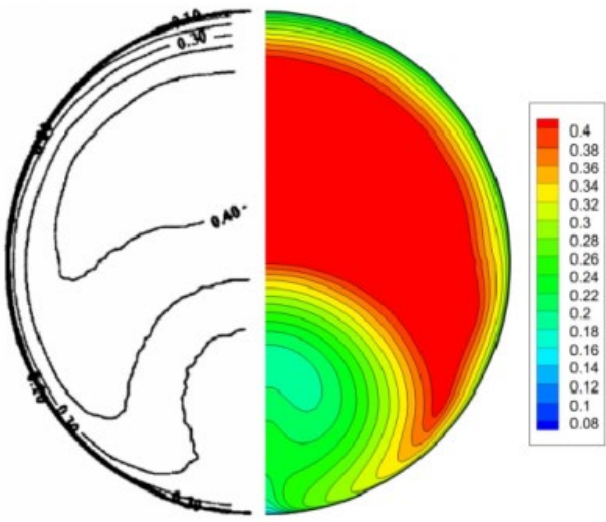

(B)

Figure 7. Mach number contours on AIP. (a) Current study contours (b) NASA Glenn experimental contours \& a previous study computational contours (Fiola, 2013)

Total pressure variation on the AIP is expressed in a form of total pressure coefficient contours as shown in Figure 8 . They turned out to be almost similar to Mach number contours on the AIP. However, the maximum value of the total pressure coefficient has slightly risen compared to the experimental one. There was also an obvious quantitative discrepancy between computational and experimental results. Experimentally, the area of total pressure deficit at the bottom of the AIP was extended above the centerline while computationally remained under the duct centerline. Figure 8 is a comparison between total pressure coefficient contours obtained computationally, and the experimental contours by NASA Glenn as well as a previous study carried out by Fiola (2013).

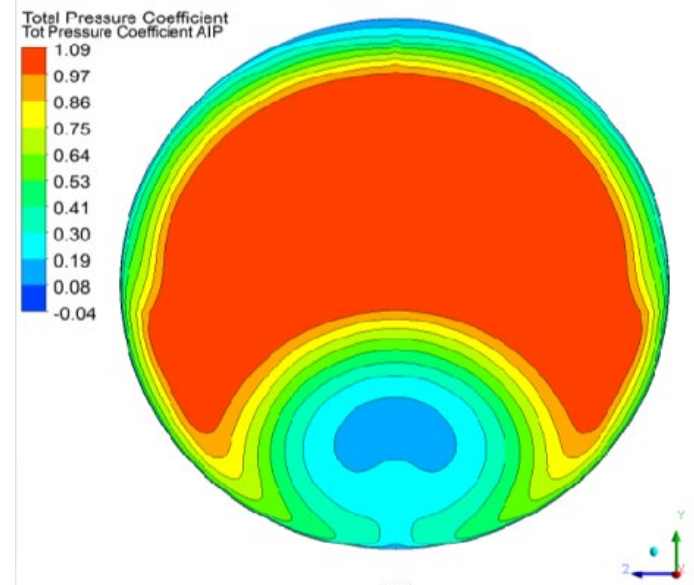

(A)
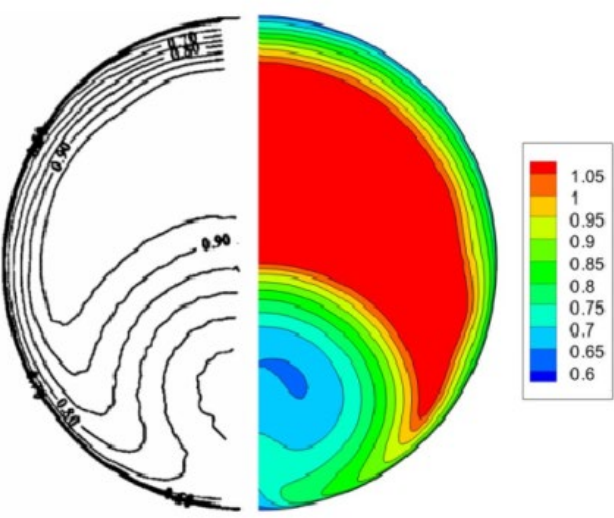

(B)

Figure 8. Total pressure coefficient contours on AIP (a) Current study contours (b) NASA Glenn experimental contours and a previous study computational contours (Fiola, 2013) 
The variation of static pressure with axial distance was measured in three different angular locations along the duct $\left(\varnothing=10^{\circ}\right.$, $90^{\circ}$, and $170^{\circ}$ ) as represented in Figure 9. The region of stream-wise separated flow is also shown. Static pressure in the three angular locations is clearly equal along the constant diameter duct attached to the geometric inlet. On the first bend of the duct, static pressure started to vary between the $\varnothing=10^{\circ}$ and the $\varnothing=170^{\circ}$ which clearly indicates the effects of streamline curvature and diffusion. Experimental results of NASA Glenn showed the separation point at $\mathrm{s} / \mathrm{D}_{1}=2.02$ and reattachment point at $\mathrm{s} / \mathrm{D}_{1}=4.13$ while current study shows that the flow starts to separate due to the reverse flow at $\mathrm{x} / \mathrm{D}_{1}=-0.103$ (experimentally corresponds to $\mathrm{s} / \mathrm{D}_{1}=2.4$ ) and reattachment occurred at $\mathrm{x} / \mathrm{D}_{1}=2.06$ (experimentally corresponds to $\mathrm{s} / \mathrm{D}_{1}=4.56$ ).

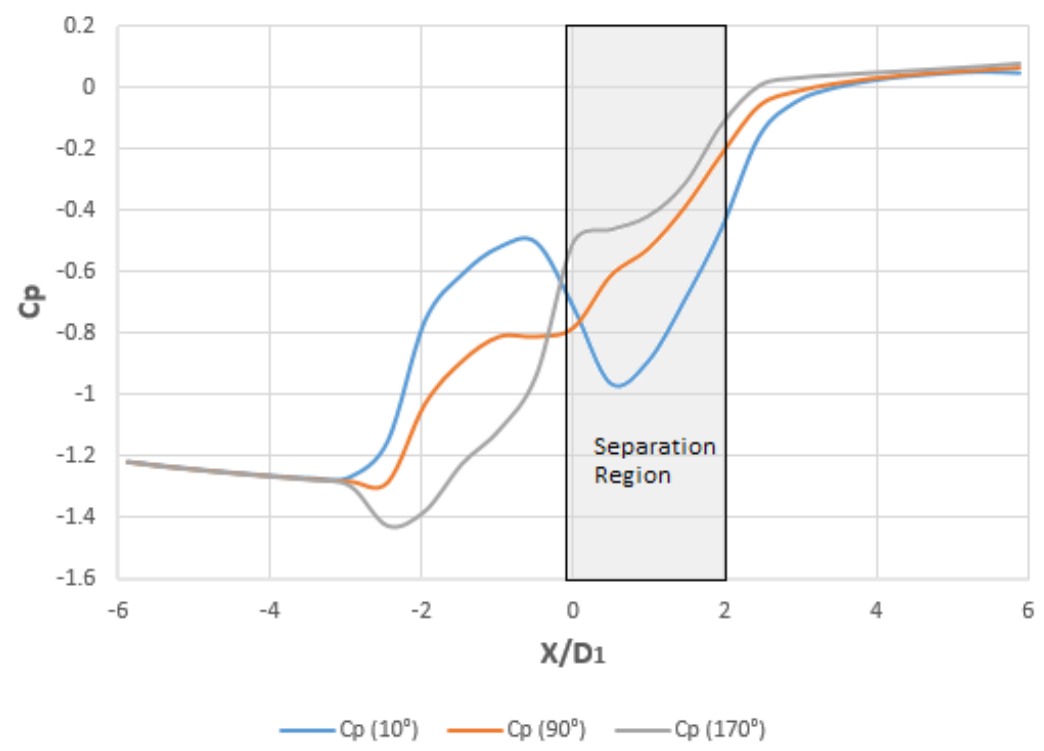

Figure 9. Surface static pressure distribution at three angular locations

\section{Jets Configuration}

5.1 Blowing jets configuration: The blowing jets configuration was initially adopted in the study based upon previous investigation carried out by Neal Harrison in his study of controlling the flow through a boundary layer ingesting S-duct. The general concept of this configuration aimed to re-energize the low-momentum flow driven by a strong secondary flow and collected at the bottom of the duct in an attempt to redistribute it along the duct periphery. Five jets were placed circumferentially at the bottom of the duct in an area of high static pressure (1.14m from the inlet). Two jets were placed at each side of the duct and one jet located exactly at the symmetry plane as shown in Figure 10. Jets incorporated only $1 \%$ the of total inlet mass flow. However, it was found that the flow distortion level was drastically exacerbated when examined on AIP. This exacerbation in the flow distortion was attributed to the contribution of the middle jets which have undesirably added more vorticity to the flow.

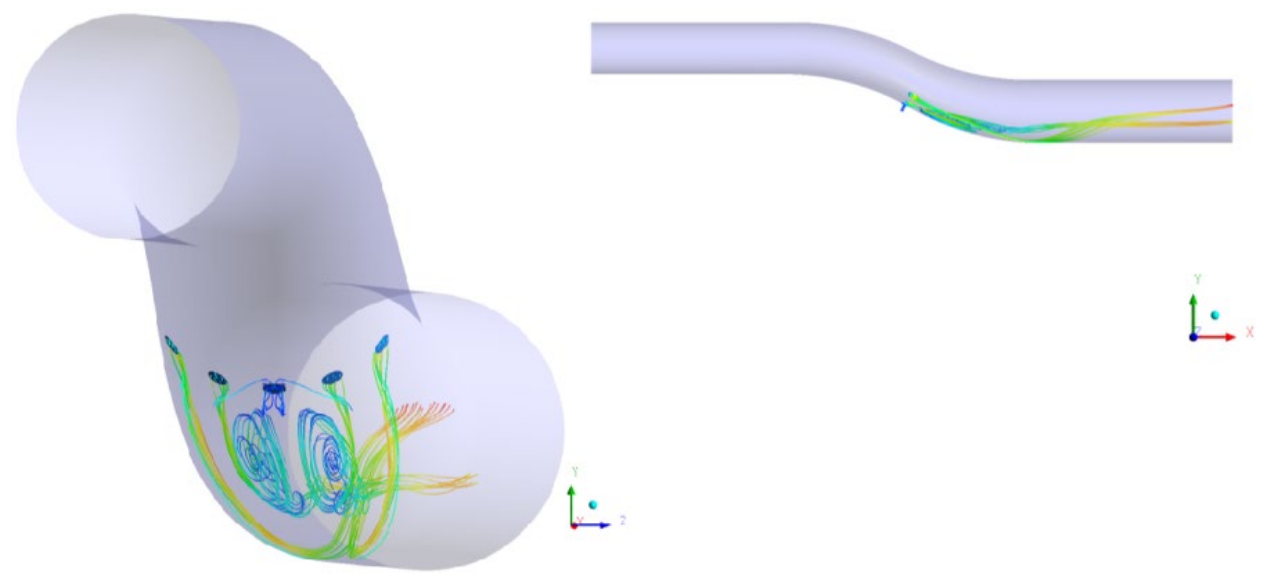

Figure 10. Blowing jets streamlines 
5.2 Suction jets configuration: As opposing to the blowing jets configuration, the concept of the suction jets adopted an idea of ingesting the swirls before their creation. Both baseline and blowing jets numerical simulations provided a good understanding of the flow field inside the S-duct. The concept behind this configuration was to address the created swirls and the secondary flow by adopting judiciously positioned suction jets. The jets were placed slightly upstream the separation onset in an area of high static pressure (first jet at $\mathrm{x}=1.24 \mathrm{~m}$ from the inlet). The jets system used only $3 \%$ of the total inlet mass flow. Jets had a circular shape with a diameter of $10 \mathrm{~mm}$. A reverse pyramid scheme was utilized as shown in Figure 11. Four jets were placed at each half of the duct while the fifth one placed exactly at the symmetry plane.

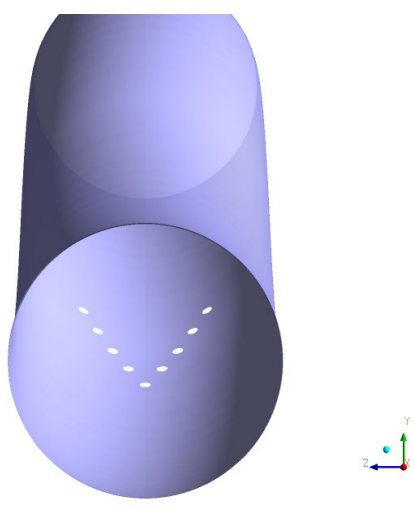

Figure 11. Reverse pyramid suction jets

\section{Flow Control Results}

The level of the pressure distortion was assessed at the AIP by comparing total pressure coefficients in both the baseline (without flow control) and the reverse pyramid scheme. The total pressure coefficient contours in Figure 12 indicate the significant impact that flow control has had on the AIP distortion. The baseline was characterized by a large region of low total pressure at the lower portion of the AIP driven by the strong secondary flow which was created due to the duct physical curvature and the crosssectional diffusion. It was obvious that reverse pyramid suction jets were able to minimize the created swirls which led to a remarkable reduction in the distorted flow at the bottom of the AIP. However, low-pressure flow increased at the top of the AIP and the periphery low momentum boundary layers became thicker. It was also noticeable that pressure recovery at the AIP undesirably affected by the suction jets. Measurements of the distortion level on the AIP were based upon using three distortion descriptors as mentioned earlier. The reverse pyramid suction scheme reduced the DC (60) $\mathrm{p}_{\mathrm{t}}$ distortion by $10 \%$ as compared to the baseline. However, jets increased the DC (60) q distortion by $9.7 \%$ compared to the baseline. The severity of the secondary flow was quantified by correlating the flow contours to the SC (60) as previously pointed out. The SC (60) showed a $16.5 \%$ reduction in the secondary flow severity.

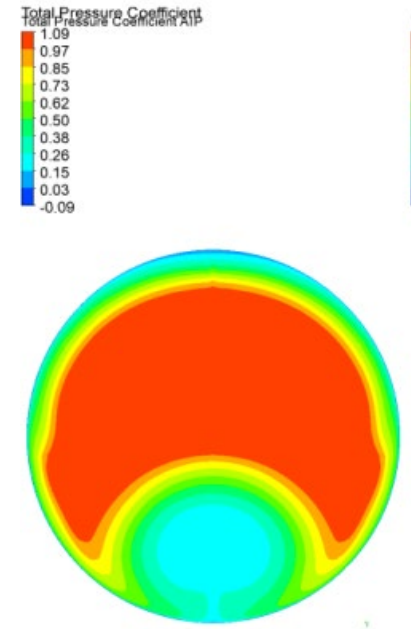

(A)
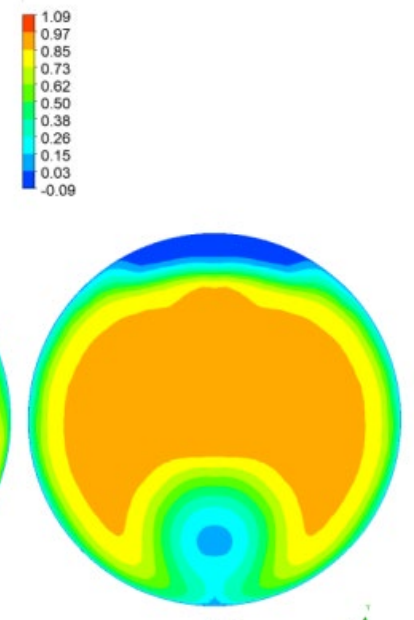

(B)

Figure 12. Comparison of total pressure coefficient (a) Baseline contours (b) Suction jets contours 
Table 2. Comparison of distortion reduction/increase on the AIP

\begin{tabular}{|c|c|c|}
\hline \multirow{2}{*}{$\begin{array}{c}\text { Distortion } \\
\text { Parameter }\end{array}$} & Baseline & Distortion Parameter Value [\% reduction/increase from baseline] \\
\cline { 2 - 3 } DC (60) $\mathbf{p}_{\mathbf{t}}$ & 0.10 & $0.09[10 \%$ reduction] \\
\hline DC (60) q & 0.82 & $0.90[9.7 \%$ increase] \\
\hline SC (60) & 0.55 & $0.46[16.5 \%$ reduction] \\
\hline
\end{tabular}

\section{Conclusions}

Baseline numerical results showed a significant agreement with the experimental test conducted in NASA Glenn research center. Incorporation of the active flow control method represented in utilizing reverse pyramid suction jets has proved its ability to address the secondary flows within the duct. Compared to the baseline, a remarkable reduction in pressure distortion and secondary flow severity was achieved on the AIP due to the suction jets. Despite the dynamic pressure increment after applying the AFC method, a $10 \%$ reduction in flow distortion achieved using DC $(60) \mathrm{p}_{\mathrm{t}}$ distortion descriptor whereas $16.5 \%$ reduction in secondary flow severity achieved using SC (60) distortion descriptor. However, pressure recovery was severely affected and a region of low pressure increased on the top of the AIP. It's highly recommended in any forthcoming study to incorporate an ejector-pump system (suction and blowing jets) so that the secondary flow within the duct would be effectively addressed. It's also expected that using an ejector-pump system and different jets schemes such as circumferential, axial, and non-reverse pyramid would result in optimally controlled flow and a significant reduction in the flow distortion on the AIP.

\section{Nomenclature}

AFC Active Flow Control

AIP Aerodynamic Interface Plane

BWB Blended Wing Body

CFD Computational Fluid Dynamics

RANS Reynold-Averaged-Navier-Stokes

UAV Uninhabited Aerial Vehicle

$C_{p} \quad$ Static pressure coefficient

$C_{p o} \quad$ Total pressure coefficient

$p \quad$ Local static pressure

$p_{o} \quad$ Local total pressure

$p_{c l} \quad$ Reference static pressure

$p_{o, c l} \quad$ Reference total pressure

$p_{t, \max } \quad$ Maximum total pressure in a six-arm distortion probe

$p_{t, \min } \quad$ Minimum total pressure in a six-arm distortion probe

$p_{t, \text { ave }} \quad$ Average total pressure in a six-arm distortion probe

$V_{C F-\max } \quad$ Maximum average secondary flow over a $60^{\circ}$ sector

$V_{C F-\min } \quad$ Minimum average secondary flow over a $60^{\circ}$ sector

$V_{\text {ave }} \quad$ Average flow velocity over a $60^{\circ}$ sector

\section{Acknowledgment}

The author expresses his appreciation to Prof. Kasu Srinivasa Reddy from Malla Reddy Institute of Engineering and Technology, Mechanical Engineering Department, Hyderabad, India for his encouragement and valuable advice.

\section{References}

Angela C. R. 2003. Effectiveness of a serpentine inlet duct flow control scheme at design and off-design simulated flight conditions. Ph. D. Thesis, Virginia Polytechnic Institute and State University. Blacksburg, Virginia, pp. 6-16.

ANSYS Fluent 13. 2015. Flow modelling software. Ansys Inc.

ANSYS ICEM CFD 13. 2014. Geometry and mesh generation pre-processor. Ansys Inc.

Aslan S., 2017. Experimental and numerical investigation of an s-duct diffuser that is designed for a micro turbojet engine powered aircraft, M.Sc. Thesis, The Graduate School of Natural and Applied Sciences of Middle East Technical University, Ankara, Turkey.

Bernard H. A. 1992. A study on vortex flow control of inlet distortion in the re-engined 727-100 centre inlet duct using computational fluid dynamics. 30th Aerospace Sciences Meeting and Exhibit. Reno, Nevada, pp. 1-4. 
Bobby L. B., Brian G. A., 2004. Experimental and computational evaluation of flush-mounted, S-duct inlets. $42^{\text {nd }}$ AIAA Aerospace Sciences Meeting \& Exhibit. Reno, Nevada, January 5-8, pp. 1-15.

Brian G. A., Lewis R. O. Numerical modelling of flow control in a boundary-layer ingesting offset inlet diffuser at transonic Mach numbers. American Institute of Aeronautics and Astronautics, pp. 1-25.

Bruce A. R., Steven R. W., Okiishi T. H., 1992. An experimental investigation of the flow in a diffusing s-duct. 28th Joint Propulsion Conference and Exhibit. NASA Technical Memorandum 105809, AIAA-92-3622 Nashville, Tennessee, July 6-8, pp. $1-10$.

Bruce A. R., Bruce J., W. 1994. Improving diffusing s-duct performance by secondary flow control. $32^{\text {nd }}$ Aerospace Sciences Meeting and Exhibit, AIAA. Reno, Nevada, pp. 1-5.

Bruce A. R., Bruce J. W. 1993. An experimental investigation of s-duct flow control using arrays of low profile vortex generators. AIAA $31^{\text {st }}$ Aerospace Sciences Meeting and Exhibit. Reno, Nevada, January 11-14, pp. 1-10.

Clinton J. F. 1972. Inlet distortion, vorticity, and stall in an axial-flow compressor. National Technical Information Service. Springfield, Vo. 22151. Monterey, California, pp. 18-25.

Fiola C.J.. 2013. Numerical simulation of separated and secondary flows in diffusing s-ducts for air breathing propulsion. Washington University in St. Louis, pp. 3-27.

Gridley M.C., Walker S.H. 1996. Inlet and nozzle technology for 21st century fighter aircraft. ASME 96-GT-244, International Gas Turbine and Aero-engine Congress. Birmingham, UK, pp. 1-3.

Giuliani J.E., Chen J.P., Beach T.A. and Bakhle M.A., 2014, Numerical simulation of boundary layer ingesting (BLI) inlet-fan interaction, $50^{\text {th }}$ AIAA/ASME/SAE/ASEE Joint Propulsion Conference. Cleveland, Ohio, pp. 1-14.

Gorton S.A., Owens L.R., Jenkins L.N., Allan B.G., Schuster E.P., 2004, Active flow control on a boundary layer ingesting inlet, $42^{\text {nd }}$ AIAA Aerospace Sciences Meeting and Exhibit, pp. 1-12

Harrison N.A., Anderson J., Fleming J.L. and Ng W.F. 2013, Active flow control of a boundary layer ingesting serpentine diffuser, Journal of Aircraft, Vol. 50, No. 1, DOI: 10.2514/6.2006-874, pp. 1-83.

Lewis R. O., Brian G. A., Susan A. G. 2008, Boundary layer ingesting inlet flow control, Journal of Aircraft, Vol. 45. No. 4, pp. 1431-1440.

Onkar S., Joseph O., Kenneth E. J., 2009, Simulation of flow control in a serpentine duct. Mechanical Aerospace and Nuclear Engineering. Rensselaer Polytechnic Institute. Troy, NY 12180, USA, pp. 1-14.

Shams M.N., Singh R.K., Zunaid M., 2016. CFD modelling of flow through s-shaped duct, International Journal of Advanced Engineering Research and Applications, Vol. 1, pp. 413-423.

Wellborn S.R., Okiishi T.H., Reichert B.A., 1993, A study of the compressible flow through a diffusing s-duct. NASA, Lewis Research Centre. NASA Technical Memorandum 106411, pp. 10-37.

\section{Biographical notes}

Yahya Shanan received B.E. in Aeronautical Engineering from Karary University, College of Engineering, Khartoum, Sudan and M. Tech. in Aerodynamics and Computational Fluid Dynamics (CFD) from Defence University, College of Engineering, Bishoftu, Ethiopia in 2011 and 2019 respectively. He works currently as a CFD freelancer. His research interests include flow distortion management.

Received September 2019

Accepted February 2020

Final acceptance in revised form February 2020 